\title{
Why cancer patients seek Islamic healing
}

\begin{abstract}
Islamic healing is frequently referred to as the treatment of choice by many Muslim cancer patients in Malaysia. Despite its widespread use, there is limited information relating to patients' healing preferences. With rising cancer rates in the country, this issue has become a concern to public health policy makers. The purpose of this study was to understand why cancer patients seek Islamic healing. This qualitative study utilized in-depth interviews with 18 cancer patients. The findings indicate three main reasons: (1) recommendations from family, friends and doctors; (2) belief in Islamic healing and (3) the perceived ineffectiveness and dissatisfaction with conventional treatments. Islamic healing will likely continue to be popular complementary cancer treatment in Malaysia as it is grounded in strong cultural and religious beliefs.
\end{abstract}

Keyword: Islamic healing; Complementary treatment; Cancer; Cancer patients 